Revista de Investigación Educativa 27

julio-diciembre, 2018 | ISSN 1870-5308 | Xalapa, Veracruz

Instituto de Investigaciones en Educación | Universidad Veracruzana

\title{
Autopercepción de la vocación en docentes de educación física escolar en Chile
}

\section{Self-perception of the vocation in school physical education teachers in Chile}

\author{
Felipe Nicolás Mujica Johnson ${ }^{a}$ \\ Nelly del Carmen Orellana Arduiz
}

Recibido: 29 de abril de 2018

Aceptado: 05 de julio de 2018

Este estudio tuvo por objetivo comprender la dinámica de la vocación, en el transcurso de la experiencia laboral de los docentes de educación física que se desempeñan en el sistema educativo escolar. La metodología utilizada corresponde al enfoque cualitativo y la participación consta de nueve docentes hombres, con edades comprendidas entre los 34 y los 62 años. El promedio de experiencia docente de los participantes es de 24 años. Los datos fueron recopilados a través de entrevistas semi-estructuradas y sometidos a un análisis de contenido deductivo-inductivo. En los resultados se presentan las siguientes familias de códigos: a) representación de la vocación docente; b) vocación por la educación física; c) factores que incentivan la vocación docente; d) factores que perjudican la vocación docente. Se concluye que la vocación docente tiene un significativo componente afectivo, que ha estimulado a los docentes a desarrollar un compromiso social durante su labor educativa.

Palabras clave: Bienestar subjetivo; experiencia pedagógica; profesión docente; educación formal; vocación.

\footnotetext{
a Doctorando en Ciencias de la Actividad Física y del Deporte. Profesor Investigador, Centro de Investigación Escolary Desarrollo, Facultad de Educación, Universidad Católica de Temuco, Chile. 凶x felipe.mujica@upla.cl

b Doctora en Filosofía y Educación. Docente del Magister de Ciencias de la Actividad y del Deporte, Facultad de Ciencias de la Actividad Física y del Deporte, Universidad de Playa Ancha de Ciencias de la Educación, Chile. 两 norellan@upla.cl
} 
The purpose of this study was to understand the dynamics of the vocation, in the course of the work experience of physical education teachers who work in the school education system. The methodology used corresponds to the qualitative approach and the participation consists of nine male teachers, aged between 34 and 62 years. The average teaching experience of the participants is 24 years. The data was collected through semi-structured interviews and subjected to an analysis of deductive-inductive content. The following families of codes are presented in the results: a) representation of the teaching vocation; b) vocation for physical education; c) factors that encourage the teaching vocation; d) factors that harm the teaching vocation. It is concluded that the teaching vocation has a significant affective component, which has stimulated teachers to develop a social commitment during their educational work.

Keywords: Subjective well-being; pedagogical experience; teaching profession; formal education; vocation.

\title{
Autopercepción de la vocación en docentes de educación física escolar en Chile
}

\author{
Self-perception of the vocation in school \\ physical education teachers in Chile
}

\section{Introducción}

T a labor docente en los sistemas educativos de nivel escolar corresponde a una Lactividad social, epistemológica y política (Rivas, Leite \& Cortés, 2014), ya que debe ser desarrollada en el marco de la legislación vigente de cada país, que ha sido construida en las sociedades democráticas por medio de múltiples diálogos que analizan las diferentes temáticas implicadas, y donde también se produce un enfrentamiento ideológico entre los diferentes sectores políticos que representan a la ciudada- 
nía. Una de las características que se atribuye a la profesión docente en América Latina es el mal reconocimiento que se le otorga en la sociedad, destacándose que cuando no existen oportunidades laborales alternativas se produce un mayor abandono de la carrera, aunque los bajos índices de ingreso a la docencia, en comparación con otras profesiones reflejan que no es una profesión atractiva (Ávalos, Cavada, Pardo \& Sotomayor, 2010). Por lo mismo, sucede que docentes de diferentes países de la región no pueden tener una dedicación completa a su labor pedagógica, ya que la situación real exige que "uno de cada tres profesores y profesoras que enseñan en escuelas primarias de América Latina complemente su labor como docente con otro trabajo" (Murillo \& Román, 2013, p. 919).

Un análisis sobre la relevancia de la educación a nivel cultural y político en México, indica que no es tema prioritario en el debate público, agregando que la docencia tendría un bajo prestigio social (Cárdenas \& Santos, 2016). En concreto, el significado de este desprestigio no enfatiza el tema de la remuneración económica, sino al "valor y sentido social que se da a la profesión docente: como profesión asalariada de segunda o la docencia como fracaso profesional” (Cárdenas \& Santos, 2016, p. 252). Esta perspectiva es coherente con un informe que aborda los estándares y la evaluación de la docencia en México, el cual plantea que el debate en torno al tema ha sido mínimo, con escasa información y por lo tanto, inefectivo, sosteniendo la esperanza de que ese documento contribuya a crear un debate público adecuado a la relevancia de su contenido (Barrera \& Myers, 2011). Esta temática fue abordada en un estudio cualitativo que contó con la participación de maestras pertenecientes a diferentes centros educativos de la Ciudad de México, en el cual se identifica que las principales problemáticas percibidas en torno al desempeño docente son el agobio laboral, la falta de actualización académica, la ausencia de vocación en el profesorado y los malos tratos recibidos de parte del alumnado o de sus padres (Fernández-Poncela, 2018).

En Colombia también se identifican dificultades para el reconocimiento social de la docencia y en particular de la concepción del docente, en comparación al de otras profesiones como la medicina. Esto ha generado que el cuerpo de docentes se encuentre realizando diversos esfuerzos para "reivindicar el estatuto de la docencia como profesión e igualmente destacar que ellos se ajustan a los estándares que rigen otras profesiones" (Osorio de Sarmiento \& Univio, 2015, p. 23). Por otra parte se encuentra un estudio realizado con el profesorado de Educación Secundaria del Departamento de Chontales en Nicaragua (uno de los países con mayor pobreza en América Latina), el cual indagó en el reconocimiento social y las condiciones laborales de la profesión 


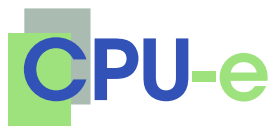

Autopercepción de la vocación en docentes

de educación física escolar en Chile

Felipe Nicolás Mujica Johnson, Nelly del Carmen Orellana Arduiz

docente, identificando que " $96.90 \%$ indica que se siente bastante o totalmente reconocido" (Zamora-Díaz, López-Noguero \& Cobos-Sanchiz, 2016, p. 197), lo cual ha sido justificado por la presencia otorgada al magisterio en los discursos políticos nacionales. Estos datos además se complementan con que " $88.60 \%$ del colectivo de profesores pertenecientes a esta muestra se siente totalmente o bastante satisfecho con su profesión" (p. 196). En cuanto a las condiciones laborales, los datos indican "que el sector del profesorado conlleva su vida y desempeño laboral en condiciones bastante precarias" (p. 197). Estos resultados permiten comprender que existe una vocación docente de carácter intrínseco que se fortalece con los logros personales y sociales, la cual se encuentra en una constante interacción con los factores de remuneración o condición laboral, pero no necesariamente serían directamente proporcionales. Por lo mismo, este último estudio concluye lo siguiente:

El profesor vivencia una profesión que, como el dios Jano, tiene dos caras que coexisten al mismo tiempo. Una es la de la satisfacción por el desarrollo de una vocación que genera muchos logros personales. Otra, la de una profesión preñada de malestares y dolencias que la sociedad no termina de percibir en su justa medida. (Zamora-Díaz et al., 2016, p. 202)

Este escenario presenta la posibilidad de que pueda existir un abuso político-institucional en torno a la vocación docente, ya que el vínculo afectivo que se desarrolla con la profesión se expresa en un compromiso social que puede convertirse en incondicional, lo que afectaría la dignidad en diferentes sentidos del profesorado. Ante esta situación, Larrosa (2010) señala que "no se puede utilizar la vocación para solicitar servicios que no corresponden al profesorado, declinar determinadas responsabilidades en los docentes ni acusarlos de la falta de vocación si no se consiguen los fines perseguidos" (p. 46).

El magisterio de Chile, principalmente el del sector público, ha participado de numerosas protestas de carácter nacional, tanto por las condiciones de agobio laboral, como por la remuneración económica, manifestando un gran rechazo al reciente sistema de desarrollo profesional docente, debido en parte a que no existe conformidad con el mecanismo utilizado para la evaluación docente. En este nuevo sistema de carrera docente, se encuentran inmersos principios de política como el aumento de la selectividad, la obligatoriedad de una evaluación diagnóstica en la formación inicial docente asociada a estándares, la inmersión profesional a cargo de un mentor y la implementación de programas para el desarrollo profesional, con el fin de establecer un ciclo de estandarización de la profesión docente (Oliva \& Gascón, 2016). El problema 
es que en estas políticas educativas no se está prestando atención a la voz del profesorado, quienes abandonan la profesión docente por motivos de insatisfacción laboral y sobrecarga de trabajo (Gaete, Castro, Pino \& Mansilla, 2017). En palabras del director de un centro educativo, se puede apreciar la falta de tiempo que tienen los docentes para preparar su evaluación, que en base a los resultados se les clasifica en diferentes tramos que son vinculantes con su remuneración:

Mi función, entonces, era apoyar, darles tiempos dentro de la jornada (por ejemplo, horas de consejo que ellos podía usar para preparar el portafolio). Por supuesto, el agobio, la angustia, el cansancio y la incertidumbre para los profesores es un lugar común. (Aravena \& Quiroga, 2018, p. 121)

Entre las problemáticas del Sistema Educativo Chileno, se encuentra la desigualdad en la calidad de la educación, asociada a las políticas de privatización que han producido una marcada segregación escolar (Cavieres, 2014; Oliva, 2008; Puga, 2011). Estas políticas educativas también se encuentran expresadas en el currículo escolar, siendo parte de "un acto con significación ideológica neoliberal, legitimando estructuras sociales, económicas, políticas y culturales hegemónicas, además de las desigualdades materiales y simbólicas que las constituyen" (Plá, 2016, p. 55). Como respuesta a este modelo educativo, surgió en el país una campaña social llamada "Alto al sistema de medición de la calidad educativa”, la cual manifiesta entre sus demandas el daño social y emocional que producen en el profesorado las exigencias por lograr buenos resultados en estas pruebas estandarizadas, entendiendo que se encuentran vinculadas a diferentes estímulos, generando dos posibles consecuencias: una recompensa económica o un castigo social (Campos-Martínez \& Guerrero, 2016). Un estudio que abordó en profundidad la perspectiva docente con respecto a la evaluación docente, evidencia el malestar que produce en el profesorado este proceso, ya que también se enmarca en un paradigma positivista que pretende estandarizar la función docente, de modo que esta sensación "se encuentra ligada a una demanda por el reconocimiento de sí. Un sí mismo que podríamos definir como una versión más auténtica y local del trabajador docente" (Fardella \& Sisto, 2015, p. 75).

En cuanto a las políticas educacionales vigentes en Chile, para optar a la profesión docente no existe ninguna normativa que haga referencia a la presencia o ausencia de vocación, como (in)habilitante para estudiar o ejercer alguna carrera pedagógica. Por lo tanto, es fundamental que el componente vocacional sea abordado tanto en los procesos de formación docente como en los de formación continua. De esta forma, se podría desarrollar o potenciar dicho componente tanto en los futuros docentes como en 
el profesorado que desempeña su labor, "mediante políticas específicas de formación y mediante dispositivos colectivos gestionados por el propio cuerpo docente" (Tenti, 2007, p. 349). Estas políticas serían consecuentes con la consideración afectiva del profesorado, ya que la presencia del componente vocacional es reconocida por favorecer el compromiso hacia la profesión (Larrosa, 2010); también, por ser "un elemento clave para atraer o distanciar al alumnado hacia el aprendizaje de una asignatura" (Bernal \& Cárdenas, 2009, p. 215). Además, este componente es muy necesario en los contextos educativos más desfavorecidos o complejos, ya que fortalece la resiliencia de los docentes (Gu \& Day, 2007, 2013), otorgándole un significado ético necesario para la excelencia laboral (Tenti, 2007). En consecuencia, un profesorado de "fuerte vocación, que cree en la potencialidad de sus alumnos y se vincula afectivamente con ellos, puede cambiar un destino que parecía adverso, al mejorar su autoestima, generar cambios en su comportamiento y en su forma de relacionarse" (Vásquez \& Halpern, 2014, p. 30).

Sobre el concepto de vocación docente, ha sido definido como una "inclinación natural para dedicarse a la actividad profesional de enseñar con entusiasmo, compromiso y confianza en el poder de la educación, dedicación especial y de servicio hacia los demás" (Larrosa, 2010, p. 49). En esta conceptualización se encuentra implícita una visión ética del profesorado, que responde a la importancia de su rol social, siendo un error creer que la vocación no debe abordarse de manera sistemática en la formación del profesorado, desde la idea de que es algo personal, subjetivo y que no tiene mayores implicaciones en el acto de educar. Como señala Fernández-Poncela (2018), las mismas maestras identifican profesionales en ejercicio de la labor docente con una ausente vocación pedagógica, lo cual podría abordarse con una perspectiva de transformación social, debido a que el componente vocacional tiene un carácter dinámico, en el sentido de que "además de construirse a través del tiempo, se construye simultáneamente en relación con ámbitos o dimensiones que influyen la tarea docente cotidiana” (Cárdenas \& Santos, 2016, p. 263). Con respecto a la decisión de ingresar a la carrera docente, un estudio con estudiantes de magisterio en España, logró identificar que "no todos los que acceden a las Escuelas de Magisterio lo hacen movidos por un sentido vocacional, ya que existen otras circunstancias en dicho acceso, que han llegado a tener un mayor peso específico" (Sánchez, 2003, p. 214).

Otro factor a considerar para este estudio es que existe un planteamiento teórico que reconoce dos tipos de vocación, una orientada a la praxis (la más deseable en educación, porque está motivada por una actividad que es valiosa en sí misma) y la segun- 
da motivada por conseguir bienes materiales o prestigio social (fines ajenos a la misma actividad) (Fuentes, 2001). A través de la presente investigación se pretenderá generar una aproximación a la vocación de carácter intrínseco que es orientada a la praxis; sin embargo, por la naturaleza del estudio se pretenderá además identificar cómo el profesorado asocia otros factores con esa vocación, los cuales pueden ser de carácter externo, como las condiciones laborales. De acuerdo con lo señalado, se plantea que si se desea avanzar hacia una calidad integral de la educación en América Latina, un eje fundamental es que la docencia sea desarrollada con una vocación orientada a la praxis, entendiendo que la tarea educativa exige una perspectiva principalmente altruista y además que se produzca una creciente dignificación de la profesión docente.

\section{Vocación a la Educación Física}

Entre las principales cualidades que debiese poseer el profesorado de Educación Física, según Carter-Thuillier y Moreno (2017), se encontrarían la "madurez y equilibrio emocional, compromiso con su función, preocupación por la persecución de fines valiosos en sí mismos, capaces de justificar sus acciones desde criterios éticos y que utilice estrategias metodológicas coherentes con esos fines" (p. 110). Estas características serían coherentes con la docencia desarrollada con vocación hacia la praxis educativa, la cual implicaría sentimientos positivos para el bienestar subjetivo, ayudando a los profesionales a afrontar las dificultades que emergen en relación al desempeño cotidiano de esta asignatura. Esta importancia de sentir agrado y autorrealización en la actividad profesional, se presentó en un grupo de docentes de Educación Física en Brasil como uno de los aspectos que les ayudó a trascender las frustraciones a lo largo de su trayectoria profesional, y a su vez, les permitió permanecer en la carrera docente hasta su jubilación (Folle \& Nascimento, 2010). Estudios cualitativos que abordan la trayectoria docente, como el mencionado con anterioridad, permiten comprender por medio de la memoria autobiográfica los sucesos más íntimos, complejos y representativos del quehacer cotidiano en el proceso educativo. Por lo mismo, se considera que ese abordaje metodológico es adecuado para indagar en la dinámica del componente vocacional de un grupo de docentes de Educación Física, ya que, como señalan Moreno, Valencia y Rivera (2016), las ideologías construidas en un cúmulo de experiencias socioculturales y pedagógicas son las que permiten conformar los significados atribuidos a la asignatura de Educación Física. 


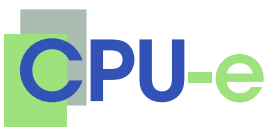

Autopercepción de la vocación en docentes

de educación física escolar en Chile

Felipe Nicolás Mujica Johnson, Nelly del Carmen Orellana Arduiz

Un estudio realizado en Chile sobre la autopercepción de la vocación docente en estudiantes de Educación Física de la Universidad de Playa Ancha en Ciencias de la Educación, identificó variados significados afectivos que justifican la decisión de haber optado por la profesión, concluyendo que la vocación se ha construido en el transcurso de las experiencias significativas presentes en sus historias de vida (Mujica \& Orellana, 2016). En concreto, en el vínculo afectivo con la profesión, destaca la seguridad, el compromiso y la felicidad por ejercerla y la posibilidad de educar por medio de la actividad física y el deporte, así como de volver a sentir las mismas emociones que en la infancia. En este estudio los estudiantes, por medio de sus narraciones, también se refirieron al rol social que debería cumplir el profesorado de Educación Física ( $\mathrm{Mu}-$ jica \& Orellana, 2016). Esta experiencia investigativa, nos revela que el ser humano no puede ser fragmentado en sus dimensiones corporales o en su historia temporal, ya que su mente inconsciente guarda mucha información del pasado, la cual podría encontrarse disponible para ser utilizada en las situaciones de la vida que lo requieran, como es el caso de decidir qué profesión estudiar o en los deseos asociados a la profesión, representando un proceso muy complejo, que refuerza la unidad que constituye a las personas. No obstante, la vocación al ser un componente dinámico, es preciso que también se indague sobre ella en los docentes durante su trayectoria profesional, ya que es bien sabido que la teoría es muy distinta a la práctica, y los estudiantes de pedagogía podrían idealizar en exceso el proceso y el contexto educativo. Específicamente, podrían ignorar que el área de la Educación Física ha sufrido históricamente una marginación frente a las otras asignaturas, en el sentido de que no siempre se le otorga la misma seriedad que requieren todas ellas, debido a que el alumnado en las clases se divierte, juega, se recrea, pero también aprende por medio de su motricidad. Producto de esto, "se tiende a categorizar a las materias según su supuesto potencial educativo. En estas categorías, la Educación Física ocupa un lugar secundario, quedando relegada a un lugar secundario frente a los aprendizajes más cognitivos" (González-Calvo, 2013, p. 87). Este asunto se ve reflejado a nivel internacional con la calidad de la educación, que ha sido reducida a objetivos de aprendizaje sobre las asignaturas de Lenguaje, Matemática, Historia o Ciencias Naturales, lo cual se consolida en los sistemas nacionales de evaluación que fomentan esa limitación conceptual (Murillo \& Román, 2010). En cuanto a las consecuencias de esta problemática, se ha señalado que ha contribuido negativamente a nivel afectivo en la experiencia de un docente novel de Educación Física cuando se ha integrado en un centro educativo (GonzálezCalvo, 2013). 
Con respecto al género y a la profesión docente en Educación Física, se puede señalar que esta asignatura, por factores histórico-culturales, ha sido estereotipada a favor de una mayoría de hombres que se identifican con el rol masculino, otorgándole las condiciones de rudeza o fortaleza características de una masculinidad hegemónica. Por este motivo, "el personal docente mantiene una visión que está influenciada por las creencias, las actitudes y los modelos hegemónicos imperantes en la sociedad” (GarcíaVillanueva, Moreno-García, Hernández-Ramírez \& Gamba-Mondragón, 2017, p. 546). Diferentes manifestaciones de estos estereotipos se pueden percibir en otros contextos asociados a la Educación Física, como es el de los roles de género que tienen las competencias deportivas a nivel internacional; tal es el caso del futbol, donde las mujeres recién en la actualidad están logrando avances en su reconocimiento deportivo. Sin embargo, en otras disciplinas como la gimnasia, el rol de la mujer es más reconocido, debido a que se caracteriza en parte por reproducir la delicadeza atribuida a ésta, que representaría a la feminidad. Precisamente dicho tema se ha estudiado en jóvenes que practican actividad física y deporte, revelándose que en la "relación deporte-masculinidad es también la percepción de las capacidades y condiciones físicas [la que se considera] como un elemento de privilegio o discriminación” (Vidiella, Herraiz, Hernández \& Sancho, 2010, p. 104). Estos roles sexistas de masculinidad y feminidad, se encuentran visibles en la clase de Educación Física (Devís, Fuentes \& Sparkes, 2005; Soler, 2009), afectando principalmente a las mujeres y a un grupo menor de hombres. No obstante, en la actualidad se observa una resistencia a este modelo y diferentes rupturas de los roles de género tradicionales (Soler, 2009). En cuanto a la labor docente, un estudio en España que consideró los roles de género en la escuela asociados a la actividad físico-deportiva, señala que una parte del alumnado reconoce que algunos docentes de Educación Física reproducen estos estereotipos, teniendo "un trato diferencial y discriminatorio hacia las chicas" (Blández, Fernández \& Sierra, 2007, p. 13). Si bien se desconocen estudios que asocien la perspectiva de género y la vocación docente en la asignatura de Educación Física, para este estudio se consideró necesario abordar un colectivo masculino de docentes, entendiendo las diferencias histórico-culturales que significan los estereotipos en la profesión. De esta forma se estaría construyendo un conocimiento que en futuras investigaciones podría ser contrastado con un colectivo del género contrario.

Por medio del presente estudio se pretende responder a la siguiente pregunta de investigación: ¿cómo perciben los docentes de Educación Física la evolución de su propia vocación en la trayectoria docente? Por lo tanto, el objetivo del estudio es comprender la dinámica de la vocación, en el transcurso de la experiencia laboral de 
algunos docentes de Educación Física que se desempeñan en el sistema educativo escolar de Chile.

\section{Metodología}

Nuestra investigación responde a la perspectiva cualitativa, la cual se caracteriza por utilizar un procedimiento dinámico y flexible (González, 2009), que permitió generar un trabajo de campo relativo a los horarios disponibles de cada docente para establecer la comunicación y aplicar una técnica de recogida de datos que se adapta al diálogo que emerge con cada participante. Además, el estudio fue orientado por el enfoque fenomenológico-hermenéutico, que se caracteriza por desvelar el significado esencial de los fenómenos y sus sentidos implícitos (Ayala, 2008). De esta forma, para comprender el fenómeno estudiado, la atención se ha focalizado en el lenguaje verbal de los participantes, ya que desde un fundamento epistemológico naturalista "el lenguaje es depositario de la historicidad del ser, la cual es indisociable del tiempo" (Fensterseifer, 2009, p. 246).

\subsection{Participantes del estudio}

Los participantes del estudio fueron nueve docentes de Educación Física, de sexo masculino y con edades comprendidas entre los 34 y 62 años. Todos los docentes al momento de participar en el estudio se encontraban desempeñando su profesión en la zona central del país, específicamente en la Región de Valparaíso y en la Región Metropolitana. El promedio de experiencia docente de los participantes es de 24 años.

Los criterios de inclusión de los participantes fueron pertenecer al sexo masculino, practicar la docencia por más de 10 años en el sistema educativo escolar de Chile y reconocer que ingresaron a estudiar la carrera por vocación a la pedagogía en Educación Física.

\subsection{Técnica de recogida de datos}

La técnica de recogida de datos seleccionada es la entrevista semi-estructurada, la cual se encuentra compuesta por un guión o guía de temas que se tratan durante una conversación (Penalva, Alaminos, Francés \& Santacreu, 2015). En este caso, el foco de 
la entrevista fue el constructo de la vocación. Algunas de las preguntas que destacan del guión, son las siguientes: a) ¿qué significa para usted la vocación?; b) ¿por qué decidió estudiar su profesión?; c) ¿cómo ha afectado su experiencia docente la vocación hacia la Educación Física?; d) ¿qué aspectos han disminuido su vocación hacia la Educación Física; y e) ¿qué aspectos han aumentado su vocación hacia la Educación Física? Las entrevistas tuvieron una duración de 40 a 60 minutos y fueron realizadas en las instituciones educativas laborales de los participantes. Para el rigor metodológico, el audio de la entrevista fue registrado por medio de una grabadora de voz, permitiendo una posterior transcripción del diálogo. La participación en la investigación fue de carácter voluntario, previa firma de un consentimiento informado, en el que se explicitó que los datos serían tratados en forma confidencial y que sus identidades se mantendrían en el anonimato.

\subsection{Análisis de datos}

Se llevó a cabo un análisis de contenido deductivo-inductivo (Osses, Sánchez \& Ibañez, 2006), en el cual las categorías o familias del estudio fueron elaboradas con anterioridad al trabajo de campo, mientras que las subcategorías o códigos emergieron con posterioridad a la recogida de los datos, en el proceso de codificación. Las familias de códigos del estudio son las siguientes: a) representación de la vocación docente; b) vocación por la educación física; c) factores que incentivan la vocación docente; d) factores que perjudican la vocación docente. El análisis se realizó por medio de tres fases tomadas de Mejía (2011); la primera consiste en la reducción de datos, la segunda, en el análisis descriptivo y la tercera, en la interpretación de los datos. En la primera fase se realizó la lectura de las entrevistas, al mismo tiempo que se fueron transcribiendo para formar los documentos primarios de la unidad hermenéutica y realizar posteriormente la codificación de los datos. La codificación corresponde a un modo sistemático de desarrollar y refinar las interpretaciones de los datos, incluyendo la reunión y análisis de todos los datos que se refieren a temas, ideas, conceptos, interpretaciones y proposiciones (Taylor \& Bogdan, 2009). La segunda fase consistió en examinar minuciosamente los datos recogidos, para construir enunciados empíricos y descriptivos del fenómeno estudiado y que respondan a las categorías pre-establecidas. Por último, en la fase final se construyeron enunciados explicativos, que constan de un respaldo de una revisión bibliográfica sobre publicaciones que se encuentran vinculadas a los resultados obtenidos, así como teorías que ayudan a la comprensión de los hallazgos, 
aportando de esta forma a la credibilidad de éstos. Para optimizar la codificación, relación y teorización de los datos, se utilizó el programa informático ATLAS.ti, versión 7.5, el cual tiene ha logrado un amplio reconocimiento por su alcance en el análisis cualitativo (Pérez-López, Morales-Sánchez, Anguera \& Hernández-Mendo, 2016). Los principales motivos para utilizar este programa informático, corresponden a las diversas posibilidades que ofrece para recuperar la información, organizarla en el proceso de codificación y asociar los diferentes códigos con las unidades significativas de una unidad hermenéutica. Para garantizar el rigor metodológico en el análisis de datos, se aplicó una triangulación de datos a nivel personal y espacial (Martínez, 2006), por medio del contraste de los hallazgos entre los diferentes sujetos (Aguilar \& Barroso, 2015), los cuales representan a la vez a diferentes espacios concretos de educación.

\section{Resultados}

El proceso de análisis de contenido generó la construcción de 13 códigos que se presentan en la Tabla 1, los cuales responden a las cuatro familias que representan las dimensiones del estudio.

\section{Tabla 1. Familias y códigos del estudio}

\begin{tabular}{|c|c|}
\hline Familias & Códigos \\
\hline \multirow{4}{*}{ Representación de la vocación docente } & Realización personal \\
\hline & Fuente de motivación intrínseca \\
\hline & Fuente de bienestar subjetivo \\
\hline & Desempeño profesional sin condiciones \\
\hline \multirow{3}{*}{ Vocación por la Educación Física } & Vínculo afectivo con el deporte \\
\hline & Educar por medio de la motricidad \\
\hline & Experiencia docente positiva en la etapa escolar \\
\hline \multirow{4}{*}{ Factores que incentivan la vocación docente } & Desarrollo integral del alumnado \\
\hline & Espacio físico del desempeño laboral \\
\hline & Amplitud didáctica de la asignatura \\
\hline & Logros profesionales \\
\hline \multirow{2}{*}{ Factores que perjudican la vocación docente } & Ausencia de valoración profesional \\
\hline & Tecnocracia del sistema \\
\hline
\end{tabular}

Fuente: Entrevistas semi-estructuradas. 
Es preciso señalar que los códigos en ningún caso representan la perspectiva global de los docentes, sino que han sido construidos con el relato individual y, en ocasiones, colectivo, lo cual se presenta en la Tabla 2, que contiene la cantidad de docentes que fundamentan cada código.

\section{Tabla 2. Fundamentación docente de los códigos emergentes}

\begin{tabular}{lc}
\hline \multicolumn{1}{c}{ Códigos } & $\begin{array}{c}\text { Fundamento personal } \\
\text { de los docente }\end{array}$ \\
\hline Vínculo afectivo con el deporte & 8 \\
\hline Desarrollo integral del alumnado & 7 \\
\hline Educar por medio de la motricidad & 6 \\
\hline Realización personal & 5 \\
\hline Fuente de motivación intrínseca & 4 \\
\hline Fuente de bienestar subjetivo & 4 \\
\hline Espacio físico del desempeño laboral & 4 \\
\hline Desempeño profesional sin condiciones & 3 \\
\hline Experiencia docente positiva en la etapa escolar & 3 \\
\hline Amplitud didáctica de la asignatura & 3 \\
\hline Logros profesionales & 3 \\
\hline Ausencia de valoración profesional & 3 \\
\hline Tecnocracia del sistema & 2 \\
\hline
\end{tabular}

Fuente: Entrevistas semi-estructuradas.

En los siguientes apartados se realiza una presentación cualitativa de los códigos, que refleja el proceso interpretativo del estudio; con el fin de aportar a la credibilidad de los hallazgos, se incorporarán citas textuales de los participantes del estudio.

\subsection{Representación de la vocación docente}

Esta familia se compone de cuatro códigos que han sido significados por los docentes de Educación Física, quienes señalan características subjetivas que componen la vocación. El primer código, realización personal, significa la coherencia que una persona 
construye entre lo que desea desempeñarse profesionalmente y lo que decide hacer. El profesorado se ha referido a conceptos como sentido de vida, llamado interior o razón de vida, para describir la importancia que le otorgan a este constructo, como expresa uno de los docentes: "la vocación es encontrar la razón de ser de tu vida, donde todo lo que haya tenga un sentido real de lo que estás haciendo" (E9).

El segundo código, fuente de motivación intrínseca, significa a la vocación como una energía que impulsa a desempeñar la labor docente, la cual se construye desde temprana edad y resulta fundamental en los momentos que se requiere enfrentar las dificultades que surgen en el campo laboral. En términos de metáfora, un docente la describió como un motor, como se señala en la siguiente frase: "el significado que le doy a la vocación, es el de un motor fundamental de lo que nos dedicamos nosotros como profesor de educación física" (E5). El beneficio de esta energía se podría percibir a diario, en la cotidianeidad del desempeño laboral, como es expresado acá: "la vocación es lo que te motiva a ser, las ganas de levantarte a trabajar en eso, todos los días de tu vida" $\left(\mathrm{E}_{3}\right)$.

El tercer código fuente de bienestar subjetivo, significa el beneficio afectivo que produce trabajar con vocación, lo cual se relaciona con el primer código de esta familia, por medio de la decisión de optar por el camino de la realización personal. En palabras de un docente, es explicado de la siguiente forma: "la vocación tiene que ser basada en los sentimientos, en el levantarse feliz para ir a trabajar, porque eso es lo que hay que hacer" (E4). En el mismo sentido, otro docente expone las consecuencias de trabajar con vocación y el bienestar que le ha proporcionado en su experiencia profesional: "cuando uno encuentra la vocación, su trabajo deja de ser algo obligado, algo desagradable, algo que tengas que hacer por obligación, pasa a ser una realidad y un placer de vida muy interesante, importante, por eso llevo 43 años trabajando en la educación" (E9).

El cuarto código, desempeño profesional sin condiciones, significa el carácter intrínseco de la vocación, debido a que no requeriría estímulos externos para que se manifieste en cada persona, de modo que un profesional que tenga la presencia de este componente en su desempeño laboral contaría con un fin en sí mismo de las actividades, como se expresa en la siguiente frase: "la vocación es una cosa interna, un interés personal de querer enseñar esta asignatura más allá del dinero que uno pueda ganar, más allá del lugar en el cual trabaje, más allá de los niños con los cuales trabaje" (E5). 


\subsection{Vocación por la Educación Física}

Esta familia se compone de tres códigos que representan los motivos que tienen los participantes para explicar su vocación por su profesión, que es la pedagogía en Educación Física. El primer código, vínculo afectivo con el deporte, significa las emociones positivas para el bienestar subjetivo que han vivenciado los docentes durante su historial deportivo, lo que les hace mencionar sentimientos que son más estables en el tiempo, como el amor al deporte, expresado en las siguientes frases: "bueno, en primer lugar yo decidí estudiar mi profesión por el amor al deporte (E1)” y “yo ingresé a la carrera porque en la época que yo estudiaba, era un amante del deporte y era la única cercanía que tenía con el deporte, la carrera de Educación Física” (E9). Otra de las formas en que han expresado el vínculo afectivo, es por medio del concepto gusto, lo cual se asocia a un tipo de placer que se vivencia con las experiencias deportivas, como se señala en la siguiente cita: "yo quise seguir la carrera de educación física porque a mí me gustaba mucho el deporte, yo lo asocié mucho por esa parte, era muy deportista y sigo siendo deportista" ( $\left.\mathrm{E}_{7}\right)$. Estos sentimientos subjetivos que se han construido en torno a la práctica deportiva, han incentivado a los participantes a expandir y transmitir los beneficios psicosociales que ellos han obtenido, como se expresará en el segundo código de esta familia.

El segundo código, educar por medio de la motricidad, significa el proyecto pedagógico que los participantes han emprendido, y uno de los principales motivos es porque ellos han sido parte de proyectos de educación motriz muy similares, siendo el agente más común el de los deportes. En la siguiente frase se puede apreciar la trascendencia que ha tenido uno de los vínculos afectivos formados a través de las experiencias deportivas personales de un docente:

El amor que uno siente por el deporte te motiva a después querer enseñarlo; al comienzo a uno le gusta ejecutar el deporte, pero después uno quiere dejar algo y la única forma es dedicándose a esta pedagogía, y poder entregar sobre todo la pasión más grande que tengo, que es por el futbol y el futbol femenino, donde he tenido grandes deportistas. ( $\left.\mathrm{E}_{1}\right)$

Al momento de la elección profesional que tienen los estudiantes al egresar de su etapa escolar, la Educación Física se presenta como una integración de dos ámbitos, el del deporte y el de la formación humana, como se expresa en la siguiente cita:

Hay dos áreas que me han acompañado en mi vida, una es el deporte y otra es el contacto con los jóvenes y con los niños. El fútbol siempre me ha acompañado 
mucho, el tenis de mesa me ha acompañado también, y en la Iglesia trabajé mucho de líder de la pastoral juvenil, lo que significaba trabajar con personas y con niños. Entonces ahí un poco nace esta necesidad de seguir de alguna forma, trabajar con niños y enseñarles actividades físicas o deportes, en este caso. Entonces ahí se asocia y la carrera que más se asemejaba a estas dos características mías que las desarrollé en la juventud era la educación física, por eso yo escogí esa carrera. (E5) El tercer código, experiencia docente positiva en la etapa escolar, significa un sentir agradable en el periodo de formación escolar durante la participación en actividades familiarizadas con la profesión, como es el caso que relata un participante:

Yo en cuarto medio tuve un taller de tenis de mesa y lo apliqué en el mismo colegio que estoy ejerciendo y siempre me he sentido muy a gusto. (E6)

\subsection{Factores que incentivan la vocación}

Esta familia se encuentra compuesta por cuatro códigos que representan a los factores que han incidido positivamente en la vocación de los docentes participantes. El primer código, desarrollo integral del alumnado, representa el desarrollo emocional, motriz y valórico que percibe el profesorado en sus alumnos, el cual reafirma su convicción acerca de la importancia de la Educación Física. En cuanto al ámbito emocional, un docente expresa lo siguiente: "lo que más me gusta es que los chicos se diviertan y que aprendan de los contenidos, que sean partícipes de su propio aprendizaje” (E2). Con respecto al ámbito motriz, se expresa de la siguiente forma: "con el pasar de los años se ve el resultado que van obteniendo mis alumnos en las clases, es gratificante ver que sus vidas han cambiado para bien, ya que llevan un estilo de vida más activo y saludable" (E8).

Por último, se ha destacado el desarrollo valórico del alumnado como un factor estimulante, lo cual se expresa en la siguiente cita:

Lo que más me gusta es el desafío permanente de tratar que los estudiantes, a través de la actividad física y del deporte, puedan educarse en base al valor, al compromiso y la responsabilidad, porque podemos desarrollar la formación integral a través de nuestra asignatura. (E8)

El segundo código, espacio físico del desempeño laboral, hace referencia al desarrollo de la asignatura en espacios abiertos y regularmente diversos. Con respecto a estas características, un docente expresa lo siguiente: "lo que más me gusta a mí es el trabajar al aire libre, trabajar en el patio, o cuando uno trabaja en el estadio. $\mathrm{O}$ sea, no todo 
trabajo tiene esa virtud de trabajar al aire libre" $\left(\mathrm{E}_{3}\right)$. Uno de los docentes explica que el trabajo en un espacio abierto o al aire libre, genera una interacción especial con el alumnado, ya que modifican la forma de expresarse que mantienen dentro de un aula cerrada, lo cual genera una buena sensación, como se señala en la siguiente cita: "el trabajar con los niños, con los jóvenes al aire libre, para mí es impagable” (E5).

El tercer código, amplitud didáctica de la asignatura, significa la diversidad de posibilidades que ofrece el currículo de Educación Física, lo cual es explicado en la siguiente frase: "la didáctica te da para muchas cosas también, hay una variable enorme de posibilidades de hacer clases al aire libre, en contacto con la naturaleza, la cantidad de deportes que uno pueda enseñar, tanto tradicionales como no tradicionales" (E5).

Por último, el cuarto, código logros profesionales, hace referencia a los resultados deseados en el desempeño de la profesión, lo cual no necesariamente se encuentra asociado a la asignatura del currículo escolar, debido a que el profesorado de Educación Física suele desempeñarse en actividades deportivas extra-programáticas, que se encuentran asociadas indirectamente a la asignatura. Estas actividades son conocidas en Chile regularmente como talleres deportivos, los cuales pueden generar diferentes tipos de logros para el establecimiento educativo; en este caso, un docente se refiere a un resultado del ámbito deportivo:

La disciplina que tienen los alumnos te permite conseguir cada proyecto que uno se propone y así logramos incluso un sueño de ser campeones de Chile, lo logramos y después nos propusimos un sueño de viajar al mundial, también lo logramos. $\left(\mathrm{E}_{1}\right)$

\subsection{Factores que perjudican la vocación}

Esta familia se encuentra compuesta por dos códigos que representan a los factores que han incidido negativamente en la vocación de los docentes participantes. El primer código, ausencia de valoración profesional, significa la percepción de un entorno que no reconoce la complejidad de la labor docente, tanto a nivel afectivo como económico. A nivel afectivo un docente señala lo siguiente: "a veces uno no se siente valorado por el entorno, ya sea por los colegas o por el colegio” (E4). Otro docente, a diferencia del anterior, percibe que esta desvalorización se manifiesta en el desinterés del alumnado:

La vocación de repente decae, porque no siempre vienen con todos los deseos de aprender y educarse, quieren el camino más corto y hoy día de repente eso no 
se ve, porque no viene bien estimulado de la familia, ni bien estimulado desde el punto de vista social. (E9)

En cuanto al factor económico, es conocido que la docencia escolar en Chile es de las peores pagadas en comparación a otras profesiones, lo cual es presentado como un aspecto que desmotiva en la siguiente cita:

De repente uno se cuestiona la carrera que uno siguió, a lo mejor porque es mal pagado. Yo creo que la parte del dinero es donde uno siempre está de por medio, pero al final gana el deseo de poder seguir trabajando, de seguir adelante y de seguir enseñando. (E6)

En cuanto al segundo código, tecnocracia del sistema, hace referencia al agobio laboral que se produce por la excesiva cantidad de documentación exigida por las autoridades técnicas del centro educativo, lo cual se expresa en la siguiente cita:

El control externo, de pronto que uno tiene que armar papeletas, que tiene que entregar documentos, que muchas veces cuesta mucho salir del colegio y hacer actividades extra programáticas, y esas cosas de cierta manera disminuyen un poco estas ganas de poder ir un poco más allá de las asignaturas que uno hace. ( $\left.\mathrm{E}_{5}\right)$

Mientras algunos docentes hacen referencia a la disminución de su vocación por algunos factores externos, otros docentes son enfáticos en señalar que la vocación no disminuye ante las dificultes y situaciones menos agradables que surgen en el sistema educativo y afectan la motivación, sino que más bien éstas son una oportunidad para incentivar la vocación, como se señala en la siguiente cita:

Las expectativas que uno tiene cuando sale de Educación Física son altas. Uno como profe dice ya, voy a enseñar esto y voy a enseñar esto otro, pero tú te das cuenta que llegas a un mundo escolar que a lo mejor le falta demasiado y tienes que potenciarlo de a poco; o sea, la vocación yo creo que no disminuye, sino que la motivación por hacer cosas, ya, pero ahí uno tiene que estar fuertemente y decir luchemos en cierto modo contra el colegio. $\left(\mathrm{E}_{3}\right)$

\section{Discusión}

En la representación social que algunos docentes han construido de la vocación docente, se ha señalado que es una fuente de motivación intrínseca, lo cual coincide con algunos significados que se le otorgan en la literatura científica, en donde se indica que "la vocación es un proceso mental que mueve a la persona desde el interior de su psi- 


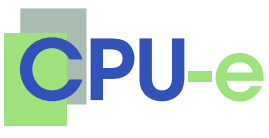

Autopercepción de la vocación en docentes

de educación física escolar en Chile

Felipe Nicolás Mujica Johnson, Nelly del Carmen Orellana Arduiz

quis o alma, es un llamado interno que lo orienta a realizar algo por más quijotesco que parece en una realidad tangible o evidente" (Franco, Vélez, López \& Becerra, 2017, p. 2). Esta vocación interna, que surge ante actividades valiosas en sí mismas, se ha clasificado como orientada a la praxis (Fuentes, 2001), la cual se asocia a otro código emergente, que es el desempeñar la profesión en forma independiente de las condiciones. Sobre el impacto que pueden tener las condiciones laborales en la vocación docente, se han identificado diferentes opiniones, ya que algunos docentes señalaron que su vocación no se ve afectada cuando las condiciones son desfavorables, mientras otros han señalado que sí se ve afectada por las condiciones. Ante esta relación, en un estudio llevado a cabo con docentes de la ciudad de Medellín, Colombia, se pudo determinar que la vocación se encuentra asociada a factores del entorno, como el obtener empleo o la posibilidad de desempeñar actividades que se encuentren al servicio de los demás (Franco et al., 2017). En relación a lo último, algunos docentes han proporcionado información que coincide con esa afirmación, ya que señalan que durante su transcurso laboral, han percibido un aumento de su vocación cuando observan progresos en el desarrollo integral de su alumnado, o cuando han podido concretar logros profesionales. De esta forma es posible entender que "cuando el docente logra satisfacer necesidades dentro de un contexto de esta naturaleza, entiende que su esfuerzo ha sido útil y verá en sus estudiantes y egresados personas que repliquen lo aprendido" (p. 6).

La vocación docente debe ser abordada desde una perspectiva sistémica, ya que es construida en un entorno social, pero en forma subjetiva a través de un proceso dinámico (Mujica \& Orellana, 2016; Villegas \& González, 2014), ocupando un rol fundamental en el proceso educativo, de modo que en la actualidad ha de entenderse como un constructo social que "se constituye con base en un proceso de elaboración continuo y socioconstructivo que impulsa a actuar en torno a determinado proyecto de vida, el cual varía en cada cultura” (Villegas \& González, 2014, p. 42). Como ha sido mencionado por parte del profesorado, la vocación es la que permite luchar contra las adversidades que surgen en el campo laboral y, por lo mismo, su opinión debe ser escuchada durante la creación de las políticas educativas locales y nacionales que se lleven a cabo, porque "todos los días ellos enfrentan la realidad escolar, por lo cual la conocen, se dan cuenta de sus necesidades y problemáticas, y entienden la urgencia de reconfigurarla a partir del compromiso social y la vocación de su misión" (Pérez, 2007, p. 112).

La identidad de cada persona se construye al mismo tiempo que la vocación, es por ello que algunos docentes la identifican como un proceso de realización perso- 
nal. En el caso del profesorado de Educación Física, ha quedado en evidencia la influencia que tuvo la identidad deportiva en el desarrollo de la vocación por su profesión, lo cual se ha integrado con una identidad de servicio público, para la formación humana. Por lo tanto, si se desea incorporar la vocación en los currículos universitarios de formación de profesorado, es fundamental que se trabaje en conjunto con el componente de la identidad, ya que "quienes acceden a la carrera docente, muchas veces, lo hacen motivados por cuestiones circunstanciales (última opción, cercanía geográfica, fracaso en otras carreras, recomendación de un amigo, entre otras)" (Villegas \& González, 2014, p. 46). Esta realidad sugiere que las instituciones de educación superior encargadas de la formación de profesores, impulsen estrategias que permitan fomentar el desarrollo de la vocación, porque tal como se ha señalado en la última cita, muchas personas pueden ingresar a la carrera docente sin vocación, pero nada les impide que puedan desarrollarla; lo mismo puede suceder en sentido contrario, que personas ingresen con vocación y la vayan perdiendo en el transcurso de su formación. En este estudio, los participantes reconocen haber ingresado a la universidad con vocación hacia la Educación Física, y luego de más de una década laboral en el sistema educativo escolar, señalan que no han perdido su vocación. Ante los múltiples casos que se pueden encontrar en la realidad educativa, es beneficioso que se realicen nuevos estudios con docentes que reconozcan estados concretos de su vocación o docentes que hayan abandonado el sistema educativo por problemas con su vocación.

La dimensión afectiva ha tenido una presencia transversal en el discurso docente, encontrándose presente en todas las categorías centrales del estudio, ya sea referida como emociones o como sentimientos que conforman el bienestar subjetivo. Con respecto a esta relación entre el afecto y la vocación, se puede mencionar que diferentes estudios (Borrachero, Dávila, Costillo \& Bermejo, 2016; Mujica \& Orellana, 2016), han demostrado que los estudiantes que se encuentran cursando carreras de pedagogía, cuando han decidido estudiar sus profesiones han utilizado como guía las emociones experimentadas en su etapa de educación escolar. En el mismo sentido, un estudio realizado en Argentina con futuros docentes pertenecientes a la Universidad Nacional de La Pampa, logró concluir por medio de sus testimonios que existe la presencia de un fuerte componente afectivo en la conceptualización de la enseñanza y del ser docente (Nosei \& Caminos, 2014, p. 67).

Entre los aspectos que pueden perjudicar la vocación hacia la Educación Física, se ha identificado el poco reconocimiento del entorno educativo sobre la labor 
docente que se realiza en esta asignatura. Éste es un factor que se ha visto también en estudios presentados en otros países, como es el caso de España, en donde se señala que en los centros educativos la asignatura de Educación Física ocupa un lugar secundario frente a las asignaturas que promueven aprendizajes principalmente de carácter cognitivo, como lengua y matemáticas, fortaleciendo una idea compartida en la comunidad educativa, que la Educación Física es irrelevante e insignificante (González-Calvo, 2013). Esta situación requiere ser analizada desde diferentes perspectivas, ya que en la actualidad puede estar generando un escenario inadecuado para el desempeño laboral de los docentes de Educación Física, más aún ante un sistema educativo que fomenta el desarrollo integral de la población escolar. Otra de las problemáticas de este código que ha sido mencionada, es la ausencia de compromiso presentada por el alumnado escolar y la falta de apoyo de la familia en estos casos, lo cual recuerda que el acto educativo se encuentra inmerso en un contexto sociocultural que no se puede desconocer y puede resultar muy desgastante para el profesorado que se enfrenta ante estas adversidades. Es sabido que el ambiente familiar es uno de los primeros entornos educativos de las personas, donde se convive en una determinada cultura moral y de hábitos sociales. Estos factores generan evidentes diferencias en la constitución psicosocial del alumnado, que en ocasiones puede ser muy afortunado en términos educativos por su contexto familiar y en otras puede ser todo lo contrario. Un estudio realizado en un sector vulnerable de Santiago de Chile (Vásquez \& Halpern, 2014), logró identificar en un centro educativo que cuenta con alumnos en situación de pobreza y riesgo psicosocial, que todo el profesorado coincide en la importancia del contexto familiar y social en la salud mental infanto-juvenil, mencionando que es uno de los problemas más frecuentes a los que se enfrentan. No obstante, el mismo cuerpo docente destaca que su vocación se encuentra orientada a ese alumnado que más lo necesita, por lo que encuentran un sentido a trabajar en ese difícil contexto pedagógico.

Entre las limitaciones de la investigación, se puede señalar que este estudio se plantea como una aproximación al fenómeno de la vocación, por lo que no se pretende establecer una explicación exhaustiva del tema; por lo demás, al ser un fenómeno que presenta una reducida producción científica, es fundamental que se construyan nuevos estudios que incorporen otros colectivos docentes, considerando diferentes aspectos geográficos, biológicos o culturales. 


\section{Consideraciones finales}

A modo de conclusión del estudio, se destaca la importancia que ha tenido la dimensión afectiva en los participantes en torno al constructo estudiado, lo cual se ha evidenciado por medio de los sentimientos que han acompañado a la vocación de los docentes de Educación Física, quienes han demostrado los deseos u objetivos que han construido en términos de logros personales y sociales. Ante esta situación, es preciso recordar que las emociones y los sentimientos subjetivos son dinámicos, por lo que requieren ser fortalecidos a través de acciones y entornos educativos propicios para un sano bienestar subjetivo. Constantemente las personas se encuentran experimentando sentimientos, y de los positivos no querrán generar un alejamiento; es por ello que los estudiantes de pedagogía en Educación Física, como los docentes participantes de este estudio, no han deseado distanciarse del ámbito deportivo, y se esfuerzan por construir un proyecto de vida que mantenga el vínculo de una u otra forma con esta práctica sociocultural. De esta manera, los docentes pretenden que sus experiencias trasciendan a otras generaciones por medio de su labor pedagógica, cumpliendo un servicio social que promueve experiencias beneficiosas para el desarrollo humano integral.

A partir de esta investigación, se pretende incitar al profesorado y a los estudiantes de pedagogía a investigar el fenómeno social de la vocación, para así ampliar su comprensión y orientar en forma más específica a los futuros maestros, tomando en cuenta el constante requisito de re-definir el perfil docente y su abordaje profesional (Martín-Romera \& García-Martínez, 2018). Los contenidos que componen los programas de desarrollo profesional docente no son neutrales, ya que responden a determinados valores o concepciones epistemológicas, los cuales son relevantes y decisivos para los estudiantes, ya que representan la base del saber con la que los futuros maestros se enfrentarán a sus prácticas profesionales (Escudero, González \& Rodríguez, 2018).

Finalmente se puede señalar que la vocación ha estimulado a los docentes a desarrollar el compromiso social durante su labor educativa, siendo vivenciada en forma subjetiva por cada participante, por lo tanto, con un significado especial para cada uno de ellos, por lo que este constructo es muy complejo de estandarizar o reducir conceptualmente, ya que representa los sentidos más profundos del ser humano. 


\section{Lista de referencias}

Aguilar, S., \& Barroso, J. (2015). La triangulación de datos como estrategia en investigación educativa. Pixel-Bit. Revista de Medios y Educación, 47, 73-88. doi:10.12795/pixelbit.2015.i47.05

Aravena, F., \& Quiroga, M. (2018). Autoetnografía y directivos docentes: una aproximación experiencial a las reformas educativas en Chile. Revista Electrónica de Investigación Educativa, 2o(2), 113-125. Recuperado de https://doi. org/10.24320/redie.2018.20.2.1600

Ávalos, B., Cavada, P., Pardo, M., \& Sotomayor, C. (2010). La profesión docente: Temas y discusiones en la literatura internacional. Estudios Pedagógicos, 36(1), 235-263.

Ayala, R. (2008). La metodología fenomenológico-hermenéutica de M. Van Manen en el campo de la investigación educativa. Posibilidades y primeras experiencias. Revista de Investigación Educativa, 26(2), 409-430. Recuperado de http:// revistas.um.es/rie/article/view/94001

Barrera, I., \& Myers, R. (2011). Estándares y evaluación docente en México: el estado del debate. Santiago: Programa de Promoción de la Reforma Educativa en América Latina y el Caribe.

Bernal, A., \& Cárdenas, A. R. (2009). Influencia de la competencia emocional docente en la formación de procesos motivacionales e identitarios en estudiantes de educación secundaria. Una aproximación desde la memoria autobiográfica del alumnado. Revista de Investigación Educativa, 27(1), 203-222. Recuperado de http://revistas.um.es/rie/article/view/94371

Blández, J., Fernández, E., \& Sierra, M. A. (2007). Estereotipos de género, actividad física y escuela: La perspectiva del alumnado. Profesorado. Revista de Currículum y Formación del Profesorado, 11(2), 1-21. Recuperado de https://www.ugr. es/ recfpro/rev112ART5.pdf

Borrachero, A. B., Dávila, M. A., Costillo, E., \& Bermejo, M. L. (2016). Relación entre recuerdo y vaticinio de emociones hacia las ciencias en profesores en formación inicial. Revista de Estudios e Investigación en Psicología y Educación, ,3(1), 1-8. Recuperado de http://revistas.udc.es/index.php/reipe/article/view/reipe.2016.3.1.723

Campos-Martínez, J. \& Guerrero, P. (2016). Efectos indeseados de la medición de la calidad educativa en Chile. La respuesta de la sociedad civil. Cadernos Cedes, 36(100), 355-374. 
Cárdenas, G., \& Santos, N. (2016). Sincronía y diacronía: una problematización de la vocación docente (segunda parte). Sincronía, 70, 244-266. Recuperado de http://sincronia.cucsh.udg.mx/portal_articulos_70/art_n7o_gco_nsl.html

Carter-Thuillier, B., \& Moreno, A. (2017). Globalización económica, postmodernidad y sistema educativo: contradicciones y alternativas desde una Educación Física crítica. Estudios Pedagógicos, 43(3), 103-117.

Cavieres, E. (2014). La calidad de la educación como parte del problema. Educación escolar y desigualdad en Chile. Revista Brasileira de Educação, 19(59), 1033-1051.

Devís, J., Fuentes, J., \& Sparkes, A. (2005) ¿Qué permanece oculto del currículum oculto? Las identidades de género y sexualidad en la Educación Física. Revista Iberoamericana de Educación, 39, 73-90. Recuperado de https://rieoei.org/historico/documentos/riezgaoz.htm

Escudero, J., González, M., \& Rodríguez, M. (2018). Los contenidos de la formación continuada del profesorado: ¿Qué docentes se están formando? Educación $X X_{1}, 21(1), 157-180$. Recuperado de http://revistas.uned.es/index.php/educacionXX1/article/view/20183

Fardella, C., \& Sisto, V. (2015). Nuevas regulaciones del trabajo docente en Chile. Discurso, subjetividad y resistencia. Psicología \& Sociedade, 27(1), 68-79.

Fensterseifer, P. (2009). Lenguaje, hermenéutica y actividad epistemológica en la Educación Física. Movimento, 15(4), 243-256.

Fernández-Poncela, A. (2018). "Ser maestra hoy y no morir en el intento”. Características y problemáticas. Revista de Educación y Desarrollo, 46, 51-60. Recuperado de http://www.cucs.udg.mx/revistas/edu_desarrollo/anteriores/46/46_FernandezPoncela.pdf

Folle, A., \& Nascimento, J. (2010). Trajetória docente em educação física: percursos formativos e profissionais. Revista Brasileira de Educação Física e Esporte, 24(4), 507-523. Recuperado de http://www.revistas.usp.br/rbefe/article/view/16785

Franco, J., Vélez, F., López, H., \& Becerra, M. (2017). Análisis de relevancia para la valoración de la vocación docente a partir de variables extrínsecas e intrínsecas: caso colegios del área metropolitana de Medellín-Colombia. Revista Espacios, $38(20), 1-7$.

Fuentes, T. (2001). La vocación docente: una experiencia vital. Revista Ars Brevis, 7 , 285-303. Recuperado de https://www.raco.cat/index.php/ArsBrevis/article/ view/90309

Gaete, A., Castro, M., Pino, F., \& Mansilla, D. (2017). Abandono de la profesión do- 
cente en Chile: Motivos para irse del aula y condiciones para volver. Estudios Pedagógicos, 43(1), 123-138.

García-Villanueva, J., Moreno-García, D., Hernández-Ramírez, C., \& Gamba-Mondragón, L. (2017). Mediciones de masculinidad y feminidad en docentes de Educación Física. Revista Internacional de Medicina y Ciencias de la Actividad Física y del Deporte, 17 (67), 541-557.

González, T. (2009). Flexibilidad y reflexividad en el arte de investigación cualitativa. Index de Enfermería, 18(2), 121-125.

González-Calvo, G. (2013). La lucha de un docente novel de educación física por el reconocimiento de su profesión narrada desde una perspectiva autobiográfica. Ágora para la Educación Física y el Deporte, 15 (2), 80-95.

Gu, Q., \& Day, C. (2007). Teachers resilience: A necessary condition for effectiveness. Teaching and Teacher Education, 23(8), 1302-1316.

Gu, Q., \& Day, C. (2013). Challenges to teacher resilience: Conditions count. British Educational Research Journal, 39(1), 22-44.

Larrosa, F. (2010). Vocación docente versus profesión docente en las instituciones educativas. Revista Electrónica Interuniversitaria de Formación del Profesorado, 13(4), 43-51. Recuperado de http://m.aufop.com/aufop/uploaded_files/articulos/1291992517.pdf

Martín-Romera, A., \& García-Martínez, I. (2018). Profesionalización del docente en la actualidad: contribuciones al desarrollo profesional. Profesorado. Revista de Currículum y Formación del Profesorado, 22(1), 7-23. Recuperado de https://recyt.fecyt.es/index.php/profesorado/article/view/63524

Martínez, M. (2006). Validez y confiabilidad en la metodología cualitativa. Paradig$m a, 27(2), 1-20$.

Mejía, J. (2011). Problemas centrales del análisis de datos cualitativos. Revista Latinoamericana de Metodología de la Investigación Social, 1(1), 47-60. Recuperado de http://www.relmis.com.ar/ojs/index.php/relmis/article/view/11/1

Moreno, A., Valencia, A., \& Rivera, E. (2016). La educación física escolar en tres centros educativos de Chile: una caracterización de sus prácticas docentes. Qualitative Research in Education, 5(3), 255-275.

Mujica, F., \& Orellana, N. (2016). Construcción de la vocación en estudiantes de Pedagogía en Educación Física: un componente subjetivo de la formación profesional. Revista de Educación Física, 34(3), 1-7.

Murillo, F. J., \& Román, M. (2010). Retos en la evaluación de la calidad de la educa- 
ción en América Latina. Revista Iberoamericana de Educación, 53, 97-120. Recuperado de https://rieoei.org/RIE/article/view/559

Murillo, F. J., \& Román, M. (2013). Docentes de educación primaria en América Latina con más de una actividad laboral. Revista Mexicana de Investigación Educativa, $18(58)$, 893-924.

Nosei, C., \& Caminos, G. (2014). Los sentimientos en la formación docente: el lugar de las pasiones instituyentes en el ejercicio de la docencia. Revista Praxis Educativa, 18(2), 62-73.

Oliva, M. (2008). Política educativa y profundización de la desigualdad en Chile. Estudios Pedagógicos, 34(2), 207-226.

Oliva, M., \& Gascón, F. (2016). Estandarización y racionalidad política neoliberal: bases curriculares de Chile. Cadernos Cedes, 36(100), 301-318.

Osorio de Sarmiento, M., \& Univio J. (2015). Vicisitudes de la formación docente en la ciudad de Bogotá. Praxis Educativa, 19(1), 20-29.

Osses, S., Sánchez, I., \& Ibañez, F. (2006). Investigación cualitativa en educación. Hacia la generación de teoría a través del proceso analítico. Estudios Pedagógicos, $32(1), 119-133$.

Penalva, C., Alaminos, A., Francés, F., \& Santacreu, O. (2015). La investigación cualitativa. Técnicas de investigación y análisis con atlas.ti. Cuenca: PYDLOS.

Pérez, D. (2007). El diario del profesor: herramienta de investigación y transformación de la práctica docente. Revista Colombiana de Rehabilitación, 6(1), 11-115.

Pérez-López, R., Morales-Sánchez, V., Anguera, M., \& Hernández-Mendo, A. (2016). Modelo tridimensional de la calidad en organizaciones deportivas: calidad emocional en usuarios/as infantiles. Cuadernos de Psicología del Deporte, 16(1), 143-150.

Plá, S. (2016). Currículo, historia y justicia social. Estudio comparativo en América Latina. Revista Colombiana de Educación, 71, 53-77. Recuperado de http://revistas.pedagogica.edu.co/index.php/RCE/article/view/4073

Puga, I. (2011). Escuela y estratificación social en Chile: ¿cuál es el rol de la municipalización y la educación particular subvencionada en la reproducción de la desigualdad social? Estudios Pedagógicos, 37(2), 213-232.

Rivas, J., Leite, A., \& Cortés, P. (2014). Formación del profesorado y experiencia escolar: las historias de vida como práctica educativa. Praxis Educativa, 18(2), 13-23.

Sánchez, L. (2003). La vocación entre los aspirantes a maestro. Educación XX1, 6, 203222. Recuperado de http://revistas.uned.es/index.php/educacion $X_{X 1 / a r t i-}$ cle/view/357 
Soler, S. (2009). Los procesos de reproducción, resistencia y cambio de las relaciones tradicionales de género en la Educación Física: el caso del fútbol. Cultura y Educación, 21(1), 31-42.

Taylor, S., \& Bogdan, R. (2009). Introducción a los métodos cualitativos de investigación. Barcelona: Paidós.

Tenti, E. (2007). Consideraciones sociológicas sobre profesionalización docente. Educação \& Sociedade, 28(99), 335-353.

Vásquez, N., \& Halpern, M. (2014). Vocación docente en profesores de una escuela vulnerable de Santiago como factor a considerar en el clima escolar y la salud mental infantil. Revista Chilena de Psiquiatría y Neurología de la Infancia y Adolescencia, 25(2), 29-38.

Vidiella, J., Herraiz, F., Hernández, F., \& Sancho, J. (octubre-diciembre, 2010). Masculinidad hegemónica, deporte y actividad física. Movimento, 16(4), 93-115.

Villegas, M., \& González, F. (2014). Narración autobiográfica: medio para construir la vocación y reconfigurar la vocación docente. Visión Sy, 12(1), 41-43.

Zamora-Díaz, W., López-Noguero, F., \& Cobos-Sanchiz, D. (2016). Realidades del empleo docente en Nicaragua. Revista Electrónica de Investigación Educativa, 18(2), 191-205. Recuperado de https://redie.uabc.mx/redie/article/ view/1127/1439 